\title{
Oral and maxillofacial surgery in China: Past, present and future
}

\author{
Jiawei Zheng $^{{ }^{*}}$, Bing Fang ${ }^{1 *}$, Shanyong Zhang ${ }^{1,2}$, Chi Yang ${ }^{1}$, Guofang Shen ${ }^{1}$, Weiliu Qiu ${ }^{1}$, \\ Zhiyuan Zhang ${ }^{1}$ \\ ${ }^{1}$ Department of Oral and Maxillofacial Surgery, Ninth People’s Hospital, College of Stomatology, Shanghai Jiao Tong University \\ School of Medicine, Shanghai, China \\ ${ }^{2}$ Shanghai Key Laboratory of Stomatology and Shanghai Research Institute of Stomatology, Shanghai, China \\ Email: braces dr@hotmail.com, Zhangshanyong@126.com
}

Received 9 August 2013; revised 9 September 2013; accepted 24 September 2013

Copyright (C) 2013 Jiawei Zheng et al. This is an open access article distributed under the Creative Commons Attribution License, which permits unrestricted use, distribution, and reproduction in any medium, provided the original work is properly cited.

\begin{abstract}
China is the most highly populated developing country. As with other scientific disciplines, dentistry (stomatology) is progressively growing since 1949 when the new China was founded. Due to the closing of the country to international discourse for several decades, international counterparts know very little about dentistry or stomatology, including the sub-discipline oral and maxillofacial surgery. This paper is aimed to introduce the scope, give a brief history, update clinical and basic research, and discuss the future of oral and maxillofacial surgery in China. It will hopefully help international colleagues to have an insight into the developmental history, education system, clinical and basic research achievements, as well as the prospective future of oral and maxillofacial surgery in China.
\end{abstract}

Keywords: Oral and Maxillofacial Surgery; Education System; China; Stomatology

\section{INTRODUCTION}

Oral and maxillofacial surgery (OMS) focuses on the prevention and treatment of diseases, injuries and deformities of the mouth, facial soft tissue, the maxillofacial bones temporomandibular joint (TMJ), salivary glands, and for certain diseases, neck pathology. OMS gradually developed into a division of general medicine, and an important part of stomatology. OMS in China includes oral and maxillofacial plastic and reconstructive surgery, orthognathic surgery, microsurgery, head and neck surgical oncology, and traditional parts of oral surgery as well which includes dento-alveolar surgery, pros-

\footnotetext{
*Bing Fang and Shan Yong Zhang contributed equally to this work.
}

thetic surgery, TMJ disease, maxillofacial trauma, salivary gland disease, etc. All these sub-disciplines are combined with traditional Chinese medicine to form OMS with Chinese features.

Due to the long time closing of the country to the international discourse, international OMS counterparts know very little about Chinese OMS. This paper is aimed to introduce the scope, give a brief history, update clinical and basic research, and discuss the future of OMS in China. The information should help international colleagues better understand the development of OMS, the educational system, clinical and basic research achievements, as well as the future prospect of Chinese OMS.

\section{BRIEF HISTORY}

A review of Chinese medical history reveals that Chinese medical scientists made important contributions to the development of OMS with respect to treating maxillofacial diseases and deformities. The Chinese medical book named Xi Jin Zhi Shu (265 to 316) provided detailed records of a particular case of cheiloplasty, including not only the patient's name, but also a detailed description of the surgical procedure and postoperative care. It is recognized as the world's first reported case of cheiloplasty. The famous American plastic surgeon, Dr. David Ralph Millard, Jr. collected this case in his monograph Cleft Craft and made various marginalia. Bei Ji Qian Jin Yao Fang (AD 652) written by Dr. Si Miao Sun of the Tang dynasty kept detailed records of the immobilization of an acute TMJ dislocation using a technique that is still in use. The approach is very scientific and corresponds with the requirements of modern anatomy and physiology. Another example of Chinese medicine is the application of acupuncture to analgesia and the treatment of Bell's palsy, which has more than 4000 years of history and has been adopted in other countries. 
The development of OMS in China is wave-like, forward spiral. Dentistry gave birth to oral surgery; maxillofacial surgery combined with oral surgery and formed a new subject called OMS. The development of modern OMS has been inextricably linked with other disciplines, such as plastic surgery, head and neck surgery, and microsurgery. At the same time, other branches were established on this basis; namely, maxillofacial plastic surgery, craniofacial surgery, maxillofacial trauma surgery, and TMJ surgery, etc. These frontier branches of medical science, in turn, promote further development of OMS.

Before 1949, diseases now in the realm of OMS were scattered within the fields of dentistry, general surgery and otolaryngology. In 1951, the former West China Union University first established an OMS ward. Also, the former Shanghai Second Medical University and the Beijing Medical University established OMS wards in 1953 and 1955. So far, more than 30 colleges of stomatology, as well as many provincial and municipal stomatological hospitals, have established independent departments and wards of OMS. Due to the destruction and interference of the Cultural Revolution in China, the first national oral and maxillofacial surgery symposium was not convened independently until 1981. In 1986, the OMS group was established and affiliated with the Chinese Stomatological Association. It held three symposia separately at Shanghai, Xi'an and Wuhan in 1986, 1990 and 1994. Since then, professional Chinese teams of oral and maxillofacial surgeons have not only been formed, but have also gradually increased in size.

Since the implement of the policy of reform and opening up to the world, Chinese oral and maxillofacial surgeons have given receptions for many of their foreign counterparts. They have also hosted international conferences of OMS, and gone abroad to participate in a variety of academic conferences around the world, giving reports and lectures to share their experiences. Since 1978, many institutions have trained hundreds of doctors majoring in OMS, and many young doctors have been sent abroad to study and do scientific research. These doctors have become the core of Chinese OMS. Chinese surgeons are active in not only dental conferences, but also the conferences of related disciplines such as: plastic and reconstructive surgery, head and neck surgery, cosmetic surgery, and other surgical disciplines. China's large population and plethora of cases provide more opportunities for practice among oral and maxillofacial surgeons, objectively. All of these factors promote the further development of OMS in China.

The development of OMS in China can be separated into three stages, namely: the initial stage, growing stage and maturity stage [1].

1) Initial stage (1950-1960): since the former Huaxi University (1951), Shanghai Second Medical University
(1953) and Beijing Medical University (1955) had separately established OMS wards, in 1956, the Ministry of Education issued a formal OMS syllabus and the first textbook Oral and Maxillofacial Surgery was edited by Professor Liang Cai Xia (1957) and published in 1965. This creation laid a solid foundation for the development of OMS.

2) Growing stage (1960-1990): the pioneers of OMS followed one after another, and made lots of exchanges to improve their level of scientific research and clinical skills. With the graduate system restored in 1978, scientific research in OMS was promoted to a higher level. The second generation of pioneers had fully taken the responsibility of boosting the development of OMS.

3) Maturity stage (since 1990): the significant effect of this stage is that OMS in China is catching up with the world's development and has won high praise. More importantly, the third-generation team of OMS has been formed, and the fourth-generation is emerging.

Among these three stages, the last 10 years of the growing stage, as well as nearly 20 years of the maturity stage, occurred 30 years after China's Reform and Opening-up, which yielded many achievements. The development and progress of OMS is a living testimony to the noteworthy achievements made by Chinese medicine after the reform and opening up to the outside world.

Considering the development of OMS in China, there are three major milestones which need to be memorized. First is the establishment of the initial OMS academic organization in 1986-the OMS Group, Stomatological Branch, Chinese Medical Association. Second, the Chinese Society of Oral and Maxillofacial Surgery, Chinese Stomatological Association formally joined the International Association of Oral and Maxillofacial Surgeons (IAOMS). Third, the $19^{\text {th }}$ International Conference on Oral and Maxillofacial Surgery (which was hosted by IAOMS and undertaken by the Chinese Society of Oral and Maxillofacial Surgery (CSOMS) and Hong Kong Society of Oral and Maxillofacial Surgery) was successfully held in Shanghai in 1999. Professor Wei Liu Qiu, member of Chinese Academy of Engineering won IAOMS's highest honorship-The Distinguished Fellow Award.

\section{EDUCATION SYSTEM}

At present, the higher education system of stomatology is divided into three different levels, namely: a 5-year, 7-year and 8-year undergraduate education system. The 5 -year education system is based on general oral medicine lessons, and a Bachelor's degree in oral medicine is granted at the students' graduation. The 7-year education system is a long-term undergraduate education, and students of this system are trained as specialists (in areas such as OMS) and are awarded the degree of Master of 
Stomatological Medicine (S.M.M) after graduation. The 8-year undergraduate education awards students the Doctor of Stomatological Medicine (S.M.D) at graduation.

According to the provisions of the National Stomatology Professional Licensing Examination, dentists must master the basic knowledge and skills of OMS in order to obtain a practicing license: 1 ) the basic knowledge and technology of OMS; 2) anesthesia and analgesia; 3) dental and alveolar surgery; 4) dental Implant surgery; 5) oral and maxillofacial infections; 6) oral and maxillofacial trauma; 7) oral and maxillofacial tumors and tumor-like lesions; 8) salivary gland diseases; 9) temporomandibular joint diseases; 10) oral and maxillofacial nerve diseases; 11) congenital cleft lip and cleft palate; 12) oral and maxillofacial imaging; 13) dentofacial malformations; and 14) oral and maxillofacial acquired deformities and defects.

OMS specialist training has not yet begun in China, even though the guideline for training and education of Chinese oral and maxillofacial surgeons [2] was published in 2006. Five-year undergraduates of stomatology can pursue the Master's degree of oral and maxillofacial surgery (3 years, $5+3$ ) after graduation, or get 3-year residency training (general oral medicine) at a general or stomatological hospital which contain a department of oral and maxillofacial surgery, according to their personal interests. After training, qualified doctors can engage in OMS clinical work. Oral and maxillofacial sur0 geons with a Master's degree may continue into a $\mathrm{PhD}$ program ( 3 years, $5+3+3$ ), which is mainly focused on scientific research. Therefore, oral and maxillofacial surgeons, in general, have graduated with: a Bachelor's from the 5-year program; a Master's from the 7-year program for Bachelor and Master Degrees in succession, or the 8-year program $(5+3)$; a Doctorate from the 8-year program for Bachelor's, Master's and Doctorate Degrees in succession or the 11-year program $(5+3+$ 3).

Colleges of stomatology in China are now offering students courses of OMS which differ from each other in teaching hours (about 120 hours on average). In order to make the teaching content more scientific, reasonable and standardized, these institutions all use the textbook Oral and Maxillofacial Surgery published by the People's Health Publishing House. The textbook, which lasted more than 30 years, has been updated to its $7^{\text {th }}$ version since publication in 1979. In 2010, the People's Health Publishing House launched Oral and Maxillofacial-Head and Neck Oncology, Oral and Maxillofacial Trauma Surgery, Orthognathic Surgery, Lip and Palate Cleft and Facial Cleft Deformity as training guide books for postgraduates of oral and maxillofacial surgery. In 2011, Chinese Society of Dental Education, Chinese
Stomatological Association organized experts to draft the Clinical Internship Standard of Oral and Maxillofacial Surgery as a reference book for colleges of stomatology. The teaching content of OMS include: lecture courses, pre-clinical laboratory practice, clinical probation and clinical internship, which each last for 12 weeks. In terms of teaching methods, in addition to the traditional theory lectures, advanced teaching concepts and methods such as problem and case based learning are also encouraged. Bilingual education and full English teaching are advocated, aiming at improving the overall quality and ability of students for future competitions.

\section{Update of Clinical and Basic Research}

China has been among the most advanced countries in the world in the clinical diagnosis and treatment of disease through OMS. In addition to routine surgeries, advanced techniques are practiced, such as: using pedicle or vascular tissue flaps to reconstruct soft and hard tissue defects, orthognathic surgery, CAD/CAM, rapid prototyping techniques, and endoscopic techniques. The Shanghai Jiaotong University School of Stomatology, Department of OMS has 230 beds, and is the largest oral and maxillofacial surgery center in Asia. It has also been designated the International Training Center for the IAOMS Oral and Maxillofacial Oncology and Reconstructive Surgery Training Fellowship Program. Together, with the Beijing University School of Stomatology, Department of Oral and Maxillofacial Surgery, both have become AOCMF international and AOCMF Reference Center-Asia Pacific. In recent years, the Department of Oral and Maxillofacial Surgery, Shanghai Jiao Tong University College of Stomatology has carried out a series of complex surgeries, such as carotid artery re- section and reconstruction, combined craniomaxillofacial resection, total glossectomy, laryngectomy and mouth floor resection and reconstruction [3]. Treatment guidelines for hemangiomas and vascular malformations of the head and neck [4] and treatment guidelines of lymphatic malformations of the head and neck were formulated and published internationally [5]. The development of OMS is highly uneven regionally, and no more than 20 hospitals are capable of diagnosing and treating extremely complex oral and maxillofacial diseases, or carrying out delicate surgeries. Most of these hospitals are affiliated with medical universities or colleges of stomatology, and $90 \%$ of these hospitals can only carry out conventional surgeries. Simple surgeries are performed by general dentists in hospitals at the county-level because there are no departments of OMS.

In the field of scientific research, China has made significant progress, whether in clinical research or basic research, compared to 20 years ago. The number of papers published in SCI by scholars of OMS has increased 
yearly, and has grown rapidly in recent years, as has their influence. But there is still a large gap when compared with the advanced countries in the world. Even though China is a populous country, there is no implant which is widely used or recognized nationally and overseas. A large quantity of advanced medical devices, equipment and reagents are dependent on imports. The most basic research often consists of follow-up studies or even repeating work of others. There is little original research in this area [6], and no work is recognized as a breakthrough in OMS by international OMS community. In clinical studies, there are no large samples or multi-center prospective studies [7]. All of these facts show that we must identify the gaps and shortcomings, and we should make unremitting efforts toward cooperation in order to yield greater achievements.

Academic journals are an important means of showing the quality of academics and research. There are two domestic professional academic journals, the China Journal of Oral and Maxillofacial Surgery and Oral and Maxillofacial Surgery. These two journals, with English abstracts, are bimonthly, and nearly 200 papers are published per year. These publications play a positive role in promoting the development of OMS by disseminating new theories, new knowledge and new technology. At present, the degree of internationalization of these two magazines is not high, and they are not included by PubMed. Since their influence is relatively limited, continuous efforts are needed to improve their academic level and the degree of internationalization. In recent years, too much emphasis was laid on the importance of SCI-cited papers, and a large number of outstanding scientific research papers were published in international journals in English. This results in problems of quantity and quality of original papers for domestic academic journals, and it is not conducive to the sustainable development of Chinese academic journals.

One measurement of the degree of internationalization of a subject is the number of specialists serving as editors of international journals. 81 Journals in Dentistry, Oral Surgery and Medicine were cited by SCI in the year of 2011. After verification, there are 11 Chinese oral and maxillofacial surgeons (including implantology) serving on the Editorial Boards of SCI cited dental journals, they are: Wei Liu Qiu (Shanghai, International Journal of Oral Science), Zhi Yuan Zhang (Shanghai, Oral Diseases), Nabil Samman, (Hong Kong, International Journal of Oral and Maxillofacial Surgery), Xu Chen Ma (Beijing, Dentomaxillofacial Radiology), Lim Kwong Cheung (Hong Kong, Journal of Oral and Maxillofacial Surgery), Song Ling Wang (Beijing, Journal of Oral Rehabilitation, International Journal of Oral Science), R. Zwahlen (Hong Kong, Oral Oncology), Jia Wei Zheng (Shanghai, Journal of Oral and Maxillofacial Surgery), Jing $\mathrm{Hu}$
(Chengdu, International Journal of Oral Science), Lian Ma (Beijing, British Journal of Oral \& Maxillofacial Surgery), Hong Chang Lai (Shanghai, Clinical Oral Implants Research). Serving as the international academic editor is not only a very important job, but also one of the hallmarks of a particular country's academic influence and status. It can also help other advanced countries in the world to appreciate the knowledge, strength, and development of OMS in China, thus increasing China's influence on international academic affairs.

\section{FUTURE PROSPECTIVE}

Currently, there are many issues to be resolved in the field of OMS. There is also a need to improve the level of prevention and treatment, especially in the fields of basic theory studies and emerging scientific technologies (molecular biology, biomedical engineering, etc.). These areas still have large gaps compared to the advanced countries throughout the world. Hard work is needed to correct this situation.

The $21^{\text {st }}$ century is an age of life science, and an age of molecular biology, which is based on the study of heredity and genetics. Diseases related to the field of OMS need the in-depth study with the help of modern technologies and methods.

The treatment style of surgical diseases in the $21^{\text {st }}$ century will change into comprehensive, sequential multidisciplinary treatment based on a team approach. The goal will be to better ensure patients' quality of life by improving patients cure rates and survival rates.

Medical science in the $21^{\text {st }}$ century has changed from pure biological treatment to a medical model combined with "environment, society, biology, psychology and engineering.” Apart from noble medical ethics and superb medical skills, doctors of OMS also need to know the art of service and the psychological demands of patients. What's more, in order to adapt to this change, doctors must learn about psychosomatic medicine and mental health.

The $21^{\text {st }}$ century is also an age of the $4 \mathrm{Ps}$ in medical science, which means preventive, predictive, personalized and participatory medicine. With the development of preventive medicine, even new infectious diseases can be better controlled. People will live longer, and the number of old people will increase. Therefore, the development of geriatrics related to OMS is inevitable.

It is an age of high technology. Advanced medical equipment brings about innovation in surgical technology. Functional surgery, minimally invasive surgery and digital surgery have become the mainstream in the development of OMS in the $21^{\text {st }}$ century. Minimally invasive surgery has been used for TMJ surgery, salivary gland surgery, neurosurgery, trauma surgery, orthog- 
nathic surgery, tumor surgery and paranasal sinus surgery, etc. With the rapid development of biomedical engineering, and the progress in regenerative medicine, especially in the studies and applications of biological materials, artificial organs (artificial teeth, artificial bone), and tissue engineering, the treatment of oral and maxillofacial surgery will be greatly influenced.

Human beings are a group, but individual differences (genetics, personality, reaction, etc.) still exist. The main task of clinical medical treatment is to focus on the individual, except in the case of group diseases. The diagnosis and treatment of individuals varies under the guidelines. With the progress in research of genes and signal transduction, digital technology (especially the application of CAD/CAM), rapid prototyping, and reverse engineering are applied in the field of OMS. "Individualized treatment" or the concepts of "custom made" and "custom design" will further improve in the $21^{\text {st }}$ century. From a molecular biology perspective, gene polymerphism will enrich disease prediction and prevention methods, and yield highly sophisticated individualized treatment regime.

In the $21^{\text {st }}$ century, medical science will pay more attention to the transformation and application of scientific achievements. Applying scientific achievements to clinical work and making an in-depth study of clinical queries in the lab are ways to achieve a virtuous circle of translational medicine [8].

\section{ACKNOWLEDGEMENTS}

This study was supported by the Third Higher Education Project in Shanghai for Undergraduates (Grant Number. 2008-73), Shanghai Jiaotong University School of Medical Education Research Projects (YB120913) and "085" Project from Shanghai Municipal Education Commission (2013).

\section{REFERENCES}

[1] Qiu, W.L. (2011) The third milestone in the development of oral and maxillofacial surgery in China. China Journal of Oral and Maxillofacial Surgery, 8, 195-198.

[2] Chinese Society of Oral and Maxillofacial Surgery (2006) Guideline for training and education of Chinese oral and maxillofacial surgeons. China Journal of Oral and Maxillofacial Surgery, 4, 304-306.

[3] Qui, W. and Zheng, J. (2003) Development of oral and maxillofacial oncology in China. Chinese Medical Journal, 116, 1567-1573.

[4] Zheng, J.W., Zhou, Q., Yang, X.J., Wang, Y.A., Fan, X.D., Zhou, G.Y., Zhang, Z.Y. and Suen, J.Y. (2010) Treatment guideline for hemangiomas and vascular malformations of the head and neck. Head Neck, 32, 10881098. http://dx.doi.org/10.1002/hed.21274

[5] Zhou, Q., Zheng, J.W., Mai, H.M., Luo, Q.F., Fan, X.D. Su, L.X., Wang, Y.A. and Qin, Z.P. (2011) Treatment guidelines of lymphatic malformations of the head and neck. Oral Oncology, 47, 1105-1109. http://dx.doi.org/10.1016/j.oraloncology.2011.08.001

[6] Zheng, J.W. (2011) On innovative study in dentistry in China. Chinese Journal of Stomatological Research (Electronic Version), 5, 115-117.

[7] Qiu, W.L. (2006) Sustained development and keep pace with the times: Prospective of development of oral and maxillofacial surgery in China. China Journal of Oral and Maxillofacial Surgery, 4, 242-245.

[8] Qiu, W.L. (2008) Oral and maxillofacial surgery. 6th Edition. People’s Medical Publishing House, Beijing. 